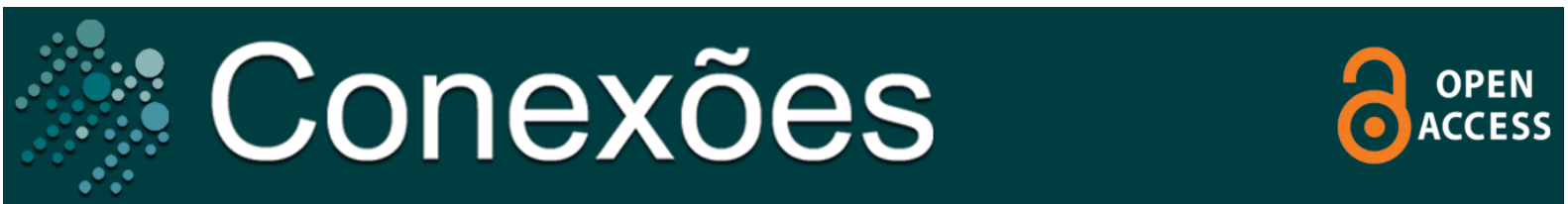

Artigo Original

\title{
Padrão de atividade física em gestantes usuárias do Sistema Público de Saúde
}

\author{
Physical activity pattern among pregnant women \\ users of the Brazilian Public Health System \\ Patrón de actividad física em mujeres embarazadas usuárias \\ del Sistema de Salud Pública
}

\author{
Carla Micheli da Silva ${ }^{1}$ \\ Daniela Saes Sartoreli ${ }^{1}$ \\ Dalmo Roberto Lopes Machado ${ }^{1}$ \\ Gleici da Silva Casto Perdoná ${ }^{1}$
}

\section{Resumo}

Objetivos: Descrever o padrão de atividade física de gestantes usuárias de Unidades Básicas de Saúde, investigar a média do gasto energético relacionados aos domínios de atividade física. Métodos: Trata-se de um estudo transversal conduzido entre 799 gestantes atendidas nas Unidades Básicas de Saúde de Ribeirão Preto, SP. A prática de atividade física foi avaliada por meio do questionário de "frequencia de atividade física diária" específico para gestantes, no qual foram obtidas a frequência, duração e o equivalentes metabólicos das atividades físicas. Resultados: A maioria das gestantes apresentaram o padrão sedentário (57\%) seguido pelo padrão leve $(43 \%)$. Em relação aos domínios de atividades físicas, o domínio doméstico teve a maior média de gasto energético $(733,7)$, enquanto que, o domínio de lazer apresentou a menor média $(20,5)$. Verificou-se que as variáveis idade acima de 30 anos, escolaridade baixa, solteira, não ser chefe de família, não trabalhar fora, e ter filhos, tiveram associações com o padrão sedentário. Conclusões: Elevada prevalência de sedentarismo foi observada entre as gestantes estudadas, sugerindo a necessidade de ações de promoção de atividade física neste público, principalmente no domínio de lazer.

Palavras-chave: Atividade física. MET, Gestação, Domínio de atividade física.

\footnotetext{
${ }^{1}$ Universidade de São Paulo, Faculdade de Medicina de Ribeirão Preto, Departamento de Medicina Social, Ribeirão Preto, São Paulo, Brasil.
}

\section{Correspondência:}

Carla Micheli da Silva. Faculdade de Medicina de Ribeirão Preto. Avenida Bandeirantes, 3900, Vila Tibério, Ribeirão Preto-SP, CEP 14049-030. Email: carlasilva@usp.br 


\begin{abstract}
Objective: To describe the physical activity pattern of pregnant women using Basic Health Units to investigate the average energy expenditure related to physical activity domains. Methods: This is a cross-sectional study conducted with 799 pregnant women attended at Basic Health Units in Ribeirão Preto, SP. The practice of physical activity was assessed using the "frequency of daily physical activity" questionnaire specific for pregnant women, in which the frequency, duration and metabolic equivalents of physical activities were obtained. Results: Most pregnant women had a sedentary pattern (57\%) followed by a mild pattern (43\%). Regarding the domains of physical activities, the domestic domain had the highest average of energy expenditure (733.7), while the leisure domain presented the lowest average (20.5). It was found that the variables: older than 30 years old, low schooling, single, not head of family, not working outside the home, and having children had associations with the sedentary pattern. Conclusions: A high prevalence of physical inactivity was observed among the studied pregnant women, suggesting the need for actions to promote physical activity in this public, mainly in the leisure domain.
\end{abstract}

Keywords: Physical activity, MET, Pregnancy, Physical activity domain.

\title{
RESUMEN
}

Objetivos: Describir el patrón de actividad física de las mujeres embarazadas que utilizan Unidades Básicas de Salud, para investigar el gasto energético promedio relacionado con los dominios de la actividad física. Métodos: Este es un estudio transversal realizado entre 799 mujeres embarazadas atendidas en las Unidades Básicas de Salud en Ribeirão Preto, SP. La práctica de actividad física se evaluó mediante el cuestionario de "frecuencia de actividad física diaria" específico para mujeres embarazadas, en el que se obtuvo la frecuencia, duración y equivalentes metabólicos de las actividades físicas. Resultados: la mayoría de las mujeres embarazadas tenían un patrón sedentario (57\%) seguido de un patrón leve (43\%). En cuanto a los dominios de las actividades físicas, el dominio doméstico tuvo el promedio más alto de gasto energético (733.7), mientras que el dominio del ocio presentó el promedio más bajo (20.5). Se encontró que las variables mayores de 30 años, baja escolaridad, soltero, no ser cabeza de familia, no trabajar fuera del hogar y tener hijos, tenían asociaciones con el patrón sedentario. Conclusiones: se observó una alta prevalencia de inactividad física entre las mujeres embarazadas estudiadas, lo que sugiere la necesidad de acciones para promover la actividad física en este público, principalmente en el dominio del ocio.

Palabras Clave: Actividad física, MET, Embarazo, Dominio de la actividad física. 


\section{INTRODUÇÃO}

Segundo a Organização Mundial da Saúde (WHO, 2010), a prática de atividade física (AF) regular está associada à redução de mortes prematuras, doenças do coração, acidente vascular cerebral, câncer de cólon e mama, diabetes tipo II entre outros. Em relação as gestantes, pode-se citar a prevenção e controle do peso, controle da taxa de glicemia, pressão arterial e da taxa de colesterol, aumento da força muscular, diminuição do stress da depressão e diminuição do parto pré-maturo (CARVALHÃES et al., 2013; TAKITO; BENÍCIO; NERI, 2009; SILVA, 2007; OLIVEIRA, 2017).

Atualmente há uma preocupação mundial crescente relacionado a qualidade e ao estilo de vida, como também em termos de consequências e efeitos nocivos à saúde causados pela inatividade. No entanto, a redução de AF é frequentemente observada na gestação motivada tanto pelas crenças populares na contradição de sua prática e nas interferências de fatores como idade, etnia, escolaridade e nível socioeconômico (OLIVEIRA, 2017).

O American College of Obstetricians and Gynecologists (ACOG) e American College of Sports Medicine (ACSM) aconselham as mulheres a realizarem AF durante a gestação. Porém, para as mulheres sedentárias antes da gestação ou para aquelas com complicações médicas ou obstétricas, devem ser avaliadas e liberadas por um médico. Estes Órgãos de Saúde recomendam 150 minutos semanais ou 30 minutos de AF diária ou quase todos os dias, de intensidade moderada, de forma contínua ou acumulada. As atividades físicas moderadas equivalem de 3 a 6 mets (equivalente metabólico), sendo que estas podem envolver atividades do dia a dia, como ir andando ao banco, escola, mercado, ao trabalho, subir escadas, varrer garagem, ou dançar (ACSM, 2000; ARTAL; O'TOOLE, 2003; ACOG, 2002).

Entretanto, a inatividade física na gestação é uma realidade tanto em países desenvolvidos como em países em desenvolvimento (CARVALHÃES et al., 2013; TAVARES et al., 2009). Domingues e Barros (2007) realizaram um estudo com 4471 mulheres na cidade de Pelotas (RS) e verificaram que antes da gravidez $14,8 \%$ das mulheres relataram alguma atividade de lazer e, apenas 4, $3 \%$ dessas mulheres mantiveram ativas durante a gestação. De acordo com Romero et al. (2015), a redução do nível de AF no período gestacional ocorre tanto em mulheres ativas anteriormente a gestação, quanto em mulheres sedentárias, as quais são poucas estimuladas a adotarem um estilo de vida ativo durante a gestação.

Dessa forma, conhecer o padrão de AF das mulheres durante a gestação é importante, uma vez que a prática regular de AF mostra ser benéfica tanto para a saúde da mãe quanto para o bebê, repercutindo positivamente sobre o baixo peso ao nascer, restrição de crescimento intrauterino, prematuridade, diabetes 
mellitus gestacional (DMG) e pré-eclâmpsia, atualmente os principais problemas em saúde materna infantil (CARVALHÃES, et al., 2013; TAKITO; BENÍCIO; NERI, 2009; OLIVEIRA, 2017). Além disso, o exercício físico durante a gestação influencia positivamente o desenvolvimento neuromotor, levando as crianças aumentarem suas habilidades motoras e assim, tornando mais ativas. Estes achados sugerem que o exercício físico na gestação pode reduzir potencialmente a obesidade infantil (MCMILLAN et al.,2019).

Assim, neste trabalho descremos o padrão de atividade física das gestantes adultas em atendimento de pré-natal em Unidades Básicas de Saúde (UBS) de Ribeirão Preto, SP e investigamos a média dos gastos energéticos das gestantes em relação aos domínios de atividade física.

\section{MÉTODO}

Os dados do presente estudo foram obtidos de uma análise secundária de um estudo transversal conduzido entre 799 gestantes adultas atendidas pelo Sistema Único de Saúde (SUS) do município de Ribeirão Preto, SP. A descrição detalhada do estudo transversal encontra-se na publicação de Barbieri et al., (2016). Este artigo segue as orientações do STrengthening the Reporting of OBservational studies in Epidemiology (STROBE) para estudos transversais.

Os critérios de inclusão do estudo foi mulheres gestantes adultas (idade $\geq$ 20 anos) e IMC pré-gestacional $\geq 20 \mathrm{~kg} / \mathrm{m}^{2}$. Foi considerada ilegível mulheres que apresentasse alguma morbidade que alterasse a atividade física habitual (insuficiência renal crônica, Síndrome da imunodeficiência adquirida (SIDA) ou câncer).

Este estudo por tratar-se de uma análise secundária o tamanho amostral baseou-se inicialmente nos dados de prevalência do DMG no Brasil. Considerando-se prevalência estimada do DMG em mulheres maiores de 20 anos usuárias do SUS no Brasil entre 5 e 10\% (BRASIL, 2010). Adotando uma margem de erro de 5\%, o tamanho amostral ideal seria de pelo menos 512 gestantes. Em termos de avaliação da AF, esse tamanho amostral é suficiente, considerando o estudo de Carvalhaes et al. (2013) que verificou a prevalência de sedentárias $50 \%$, com intervalo de $90 \%$ de confiança e precisão de $5 \%$, resultou em 253 gestantes.

A AF foi avaliada a partir do questionário nominado de "Questionário de Frequência de Atividade Física Diária" (QAFD) previamente desenvolvido e validado para gestantes brasileiras (TAKITO, NERI, BENÍCIO, 2008). Assim, a frequência (semanal) e a duração (minutos) das atividades físicas foram obtidas por meio do QAFD e, posteriormente, utilizando o Compêndio de Atividade Física (AINSWORTH et al., 2000), verificou-se o MET (equivalente metabólico) 
referente a cada AF realizada pelas gestantes na semana. Portanto, a AF inclui além dos exercícios físicos, os domínios das atividades físicas cotidianas como: doméstico, ocupacional, de locomoção e de lazer. Sendo assim, com objetivo de englobar todas as atividades do cotidiano das participantes, as atividades físicas foram divididas de acordo com os domínios de AF em: doméstico, ocupacional, de locomoção, de lazer e inatividades. Dessa forma, considerou-se como atividades domésticas todas as atividades cotidianas, realizadas no domicílio, tais como: limpeza leve, faxina, varrer casa, varrer quintal, limpar tapete ou carpete, lavar roupa, estender roupa no varal, passar roupa, cozinhar, lavar louça, cuidar de criança, brincar com criança, carregar criança no colo. Consistiram atividades ocupacionais todas aquelas realizadas no contexto profissional como: ficar sentada ou em pé no trabalho (GUSTAVO, 2010; CHASAN-TABER, 2004). No domínio locomoção foram consideradas as caminhadas no sentido de se deslocar de um lugar para o outro, como por exemplo: ir a pé de casa para mercado, padaria, buscar criança na escola e fazer compras. No domínio de lazer considerou-se exercício físico e atividade sexual e no grupo da inatividade todas as atividades sedentárias.

Para o cálculo do padrão de AF das gestantes utilizou-se a equação previamente desenvolvida por Tavares et al. 2009 em que propuseram criação de um MET geral para cada gestante, denominado "METgest". Assim, cada gestante temos o valor do METgest dado por:

$$
\text { MetGest }_{i}=\sum_{j=1}^{k} \frac{\text { frequência }_{k} X \text { Tempo }_{k} X \text { Met }_{k}}{\sum_{j=1}^{k} \text { Frequencia semanal }_{k} X \text { Tempo semanal }_{k}}
$$

onde TTSP é o Tempo Total Semanal Ponderado de cada atividade. O TTSP é obtido pelo Tempo Total Semanal (TTS) multiplicado pelo índice do MET da késima atividade. $\mathrm{O} T T S_{k}$ é obtido pelo produto da Frequência de realização semanal pela duração da atividade $(T T S=F \times T)$ para cada atividade.

Posteriormente o METgest foi separado de acordo com os domínios de AFs gerando um nível de intensidade para cada gestante, podendo, portanto, classificá-las como gestante com padrão de AF sedentário ( $<1,5$ METs), leve ( 1 , 5-2, 9 METs), moderado (3, 0-5, 9 METs) e intenso ( $\geq 6,0$ METs) (CHASANTABER, 2004).

As características sócio-demográficas, estado nutricional e estilo de vida, foram coletadas através de um questionário estruturado. O questionário abrangia questões referentes a: idade, em anos, e cor da pele referida pela gestante, sendo está classificada como: branca, parda e outras. O estado civil foi dividido em duas categorias, casada ou solteira, separada ou viúva, tendo como base a presença ou não de companheiro. A escolaridade da gestante foi coletada em anos de estudo. O questionário apresentava também questões relacionadas à 
saúde prévia das gestantes como: tabagismo (nunca fumou, parou ao engravidar ou fuma atualmente), histórico familiar de DMG, diabetes prévia, hipertensão pré-gestacional (sim/não). E outras questões como: estrato social, trabalha fora de casa (sim ou não), renda per capita, índice de massa corporal (IMC) prégestacional e atual $\left(\mathrm{kg} / \mathrm{m}^{2}\right)$, peso pré-gestacional e peso atual, números de filhos, consultas pré-natal, semana gestacional até o momento da entrevista e estado nutricional. Para a classificação do estrato socioeconômico, foi utilizado o Critério de Classificação Econômica Brasil (ABEP, 2015), baseado na posse de itens e no grau de instrução do chefe da família.

Para o cálculo da idade gestacional foi considerada a data da última menstruação (DUM) registrada no cartão da gestante. O peso pré-gestacional foi referido pela gestante e confirmado pelo cartão da gestante. Medidas de peso $(\mathrm{kg})$ e estatura $(\mathrm{m})$ foram obtidas em balança digital (TANITA modelo HS302) e estadiômetro portátil (SANNY modelo ES2040), respectivamente. O IMC prégestacional e o atual foram obtidos pela divisão do peso pré-gestacional e atual divididos pela altura ao quadrado. Para a adequação do IMC pré-gestacional foram adotados os critérios do Institute of Medicine (1990). Para a adequação do IMC segundo a semana gestacional foram empregados os critérios propostos por Atalah (1997).

Estas variáveis foram categorizadas para comparação com a variável referente, o padrão de atividade física (sedentário e leve). O TTSP e o TTS foram considerados variáveis contínuas.

Os questionários aplicados foram revistos, codificados e inseridos em banco de dados específico, em programa Excel ${ }^{\circledR}$. Na análise estatística calculou-se a frequência das variáveis categóricas e a média e Desvio Padrão (DP) das variáveis contínuas. Para verificar associação entre as variáveis categóricas, com o padrão de $A F$, utilizou-se o teste Qui-quadrado e a odds ratio (OR) como medida de efeito. Para verificar se houve diferenças entre os grupos das variáveis contínuas utilizou-se o teste $\mathrm{t}$ de Student para comparação de duas médias e ANOVA para médias ou equivalentes não paramétricos. Optou-se pelo teste Kruskal Wallis por ser um teste não paramétrico utilizado para comparar três ou mais populações. O nível de significância considerado foi de $a=5 \%$ e as análises estatísticas foram realizadas com o auxílio do programa estatístico Stata versão 12.0 e R Cran (R. CORE TEAM, 2.013).

O estudo foi aprovado pelo Comitê de Ética em Pesquisa do Centro de Saúde Escola da Faculdade de Medicina de Ribeirão Preto, da Universidade de São Paulo (277/10/COORD.CEP/CSE-FMRP-USP). As gestantes que concordaram em participar do estudo assinaram o Termo de Consentimento Livre e Esclarecido. 


\section{Resultados E Discussão}

As entrevistadas apresentaram uma média (DP) da idade de 27, $65(5,4)$ anos, com a maioria (66\%) na faixa etária de 20 a 30 anos, enquanto que a média (DP) da escolaridade foi de $8,7(3,2)$ anos de estudos. As gestantes desse estudo eram na sua maioria primigestas (65\%), casadas (78\%), não trabalhava fora de casa (53\%). Um pouco acima da metade (53\%) estavam com peso adequado antes da gestação, de acordo com a adequação do IMC prégestacional, porém, quando considerou a adequação do IMC segundo a semana gestacional, o percentual de mulheres com peso adequado diminui para $39 \%$, aumentando o número de gestantes com sobrepeso (34\%) e obesas $(24 \%)$. (TABELA 1).

Tabela 1 - Características sociodemográficas, estado nutricional e estilo de vida das gestantes, Ribeirão Preto / SP, 2020

\begin{tabular}{|c|c|c|c|}
\hline \multirow{2}{*}{ Características } & & $\mathrm{N}=799$ & $\%$ \\
\hline & $20-29$ & 533 & 66 \\
\hline \multirow[t]{2}{*}{ Idade (anos) } & $30-45$ & 266 & 34 \\
\hline & $<8$ & 271 & 30 \\
\hline \multirow[t]{3}{*}{ Escolaridade (anos) } & 8 a 11 & 466 & 60 \\
\hline & 12 e + & 62 & 10 \\
\hline & $A+B$ & 158 & 19 \\
\hline \multirow{3}{*}{ Classe social* } & C & 535 & 67 \\
\hline & $D+E$ & 106 & 14 \\
\hline & Marido & 557 & 70 \\
\hline \multirow[t]{2}{*}{ Chefe de família } & A própria gestante & 106 & 13 \\
\hline & Outros & 136 & 17 \\
\hline \multirow{3}{*}{ Estado Civil } & Solteiras & 170 & 22 \\
\hline & Casadas & 629 & 78 \\
\hline & Não trabalha & 430 & 53 \\
\hline \multirow[t]{2}{*}{ Trabalho fora de casa } & Domésticas & 64 & 8 \\
\hline & Outros & 305 & 39 \\
\hline \multirow{3}{*}{$\begin{array}{l}\text { Adequação do IMC** } \\
\text { pré- gestacional }^{+}\end{array}$} & Peso adequado & 423 & 53 \\
\hline & Sobrepeso & 225 & 28 \\
\hline & Obesidade & 143 & 19 \\
\hline \multirow{4}{*}{$\begin{array}{l}\text { Adequação do IMC** } \\
\text { segundo da semana } \\
\text { gestacional }++\end{array}$} & Baixo peso & 31 & 3 \\
\hline & Adequado & 314 & 39 \\
\hline & Sobrepeso & 267 & 34 \\
\hline & Obesidade & 193 & 24 \\
\hline \multirow{2}{*}{ Tem outros filhos } & Não & 275 & 35 \\
\hline & Sim & 522 & 65 \\
\hline \multirow{2}{*}{$\begin{array}{l}\text { Sentimento de } \\
\text { tristeza }\end{array}$} & Sempre & 319 & 40 \\
\hline & As vezes & 480 & 60 \\
\hline Pressão Alta & Sim & 424 & 53 \\
\hline \multirow{2}{*}{$\begin{array}{l}\text { Sono tranquilo até o } \\
\text { momento da } \\
\text { entrevista }\end{array}$} & Sim & 94 & 11 \\
\hline & Não & 705 & 89 \\
\hline
\end{tabular}

* ABEP (2015); ** IMC (índice de massa corpórea; + de acordo com os critérios de Institute of Medicine (1990); ++ de acordo com Atalah (1997) 
Em relação ao padrão de $A F$ verificou-se predominante o padrão sedentário (metgest $<1,5)$ seguido pelo padrão leve $(1,5 \leq$ metgest $<3,0)$. Os padrões moderado e intenso não foram observados em nenhuma das gestantes desse estudo (Tabela 2).

Tabela 2 - Classificação das gestantes segundo o padrão de atividade físicaRibeirão Preto/SP, 2020

\begin{tabular}{lcc}
\hline \multicolumn{1}{c}{ Padrão de $\mathrm{AF}^{*+}$} & $\mathrm{N}$ & \% \\
\hline Sedentário & 457 & 57 \\
Leve & 342 & 43
\end{tabular}

*AF (atividade física); ${ }^{+}$Classificação segundo Chasan-Taber et al. (2004).

Em relação a variável TTSP a tabela 3 apresenta a média do TTSP dos domínios por semana e a figura 1 a média do TTSP dos domínios por dia. Ambos mostram que o domínio doméstico teve a maior média, seguido pelo domínio ocupacional e pela inatividade. Os domínios de lazer e de locomoção apresentaram as menores médias de TTSP, respectivamente (Tabela 3 e Figura 1). Em termos médios, o domínio doméstico e de ocupação são próximos quando abordamos o periodo de um dia, mostrando que o tempo gasto com esta atividade são similares.

Tabela 3: Média e desvio padrão do TTSP segundo os domínios de atividade física das participantes do estudo - Ribeirão Preto/SP, 2020

\begin{tabular}{cc}
\hline Domínios & TTSP* \\
& Média (DP) \\
\hline Doméstico $(\mathrm{N}=782)$ & $733,7(578,7)$ \\
Ocupacional $(\mathrm{N}=346)$ & $718,7(324,4)$ \\
Locomoção $(\mathrm{N}=555)$ & $52,5(69,4)$ \\
Lazer $(\mathrm{N}=580)$ & $20,5(34,8)$ \\
Inatividade $(\mathrm{N}=798)$ & $625,8(182,7)$ \\
Total $(\mathrm{N}=798)$ & $1143,1(320,8)$ \\
\hline
\end{tabular}

${ }^{*} T \mathrm{SP}=$ Frequência da atividade física $X$ Duração em minutos por semana da atividade física $X$ MET da atividade física. 


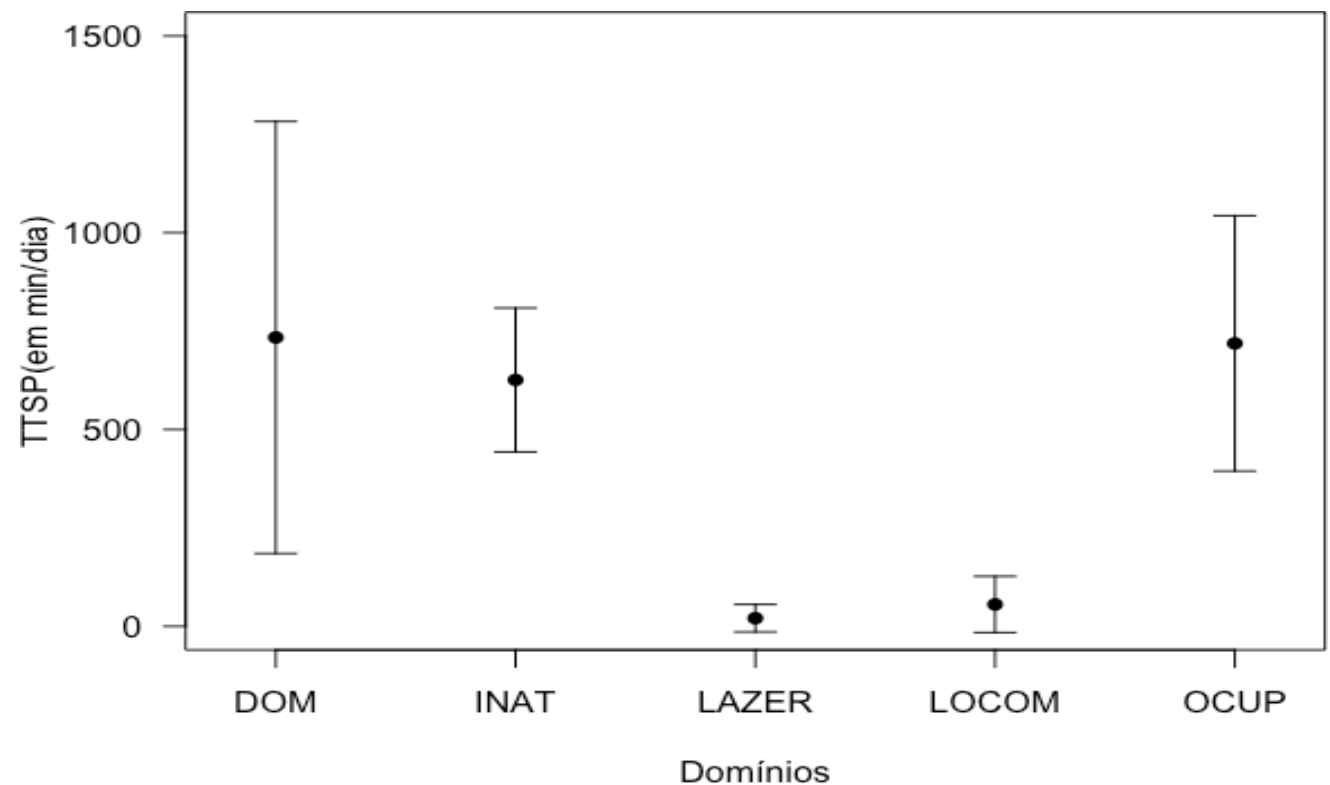

Figura 1 - Média e Desvio padrão (+ e -) do TTSP em minutos/dia para os diferentes Domínios. $* T T S P=$ Frequência da atividade física $X$ Duração em minutos da atividade física $X$ MET da atividade física; DOM= Doméstico; INAT= Inatividade; LOCOM= locomoção; OCUP= ocupação.

Considerando a associação do padrão de AF com as variáveis do estudo, verificamos que gestante com idade superior a 30 anos, que eram chefe de família, casada, com escolaridade superior a 12 anos, primigesta e que trabalhavam fora de casa, apresentaram menor probabilidade de terem o padrão sedentário. As demais variáveis não apresentaram associação com o padrão de AF. (Tabela 4)

Tabela 4 - Características das gestantes de acordo com o padrão de atividades físicas - Ribeirão Preto/SP, 2020

\begin{tabular}{|c|c|c|c|c|c|}
\hline Variáveis & Leve & Sedentário & Total & OR $(95 \%)$ & Valor $\mathrm{p}^{*}$ \\
\hline Idade & N (\%) & $N(\%)$ & $\mathrm{N}$ & & \\
\hline $20-25$ & $82(32)$ & $182(68)$ & 264 & - & \\
\hline $25-30$ & $123(45)$ & $147(54)$ & 270 & $0,57(0,40 ; 0,8)$ & \\
\hline $30-35$ & $77(46)$ & $89(53)$ & 166 & $0,55(0,37 ; 0,8)$ & $<0,001$ \\
\hline $35-40$ & $40(54)$ & $34(45)$ & 74 & $0,41(0,24 ; 0,6)$ & \\
\hline $40-45$ & $16(64)$ & $9(36)$ & 25 & $0,27(0,11 ; 0,6)$ & \\
\hline \multicolumn{6}{|l|}{ Escolaridade } \\
\hline$>0$ a 4anos & $29(48)$ & $32(52)$ & 61 & - & \\
\hline 9 a11 anos & $186(40)$ & $280(60)$ & 466 & $1,30(0,79 ; 2,3)$ & \\
\hline 5 a 8 anos & $104(50)$ & $104(50)$ & 208 & $0,90051 ; 1,60)$ & 0,04 \\
\hline Até 4 anos & $22(35)$ & $42(65)$ & 64 & $1,62(0,82 ; 3,4)$ & \\
\hline \multicolumn{6}{|l|}{ Chefe de Família } \\
\hline Marido & $303(45)$ & $349(55)$ & 652 & - & \\
\hline Própria & $55(53)$ & $47(46)$ & 10 & $0,72(0,47 ; 0,98)$ & \\
\hline Pai/Mãe/outro & $30(22)$ & $107(78)$ & 137 & $3,01(1,90 ; 4$ & $<0,001$ \\
\hline $\begin{array}{l}\text { S } \\
\text { Estado civil }\end{array}$ & & & & & \\
\hline Solteira & $55(32)$ & $116(67)$ & 171 & - & $<0,001$ \\
\hline Casada & $286(45)$ & $342(55)$ & 628 & $0,57(0,40 ; 0,81)$ & \\
\hline
\end{tabular}




\begin{tabular}{|c|c|c|c|c|c|}
\hline \multicolumn{6}{|c|}{ Trabalho } \\
\hline Sim & $204(54)$ & $173(46)$ & 377 & - & \multirow[t]{2}{*}{$<0,001$} \\
\hline Não & $137(32)$ & $285(67)$ & 422 & $\begin{array}{c}2,43(1,82 ; 3, \\
28)\end{array}$ & \\
\hline \multicolumn{6}{|c|}{ Tem outros filhos } \\
\hline Sim & $68(23)$ & $212(76)$ & 280 & - & \\
\hline Não & $274(53)$ & $245(46)$ & 519 & $\begin{array}{c}0,26(0,19 ; 0, \\
37)\end{array}$ & $<0,001$ \\
\hline
\end{tabular}

$*_{p}<0,05$, Teste Qui quadrado

O presente estudo verificou a prevalência de gestantes com o padrão sedentário seguido pelo padrão leve, sendo que os padrões moderados e intensos não foram observados. Em relação à média de gasto energético (TTSP) nos domínios de $A F$, os domínios de lazer ter a menor média, enquanto que o domínio doméstico a maior média de TTSP. Considerando as associações das variáveis com o padrão de $A F$, verificamos associações positivas com o padrão sedentário, ter idade menor que 30 anos, escolaridade menor que 4 anos, ser solteira, não ser chefe da família, não trabalhar fora de casa e outros filhos.

Resultados similares foram encontrados em dois estudos (TAVARES et al., 2009; BICALHO, 2006) ambos verificaram uma prevalência de quase todas as gestantes sedentárias, não tendo sido observada nenhuma gestante com o padrão de AF moderado ou intenso. Já o estudo de Carvalhaes et al. (2013), verificou também uma alta prevalência de gestantes sedentárias (77\%), porém, encontraram mulheres com o padrão moderado $(12,5 \%)$ e intenso $(9,8 \%)$. 0 estudo de Schimit et al. (2006) realizado nos Estados Unidos (EUA), observou; um predomínio do padrão sedentário e baixa prevalência do padrão moderado, mesmo em mulheres com melhores condições socioeconômicas e maior nível educacional do que as gestantes brasileiras atendidas pelo SUS. Dessa forma, observa-se que tanto em países desenvolvidos como em países em desenvolvimento, apesar das diferenças socioeconômicas e culturais, o comportamento das mulheres durante a gestação em relação à AF é semelhante (CARVALHÃES et al., 2013; TAVARES, 2009).

Para alguns autores, se a gestante é saudável, esse comportamento sedentário, parece estar associada a um comportamento cultural (OLIVEIRA et al., 2017). Outros fatores que podem influenciar esse comportamento incluem a orientação e o apoio dos profissionais de saúde. Estudo mostra que a orientação fornecida pelos profissionais de saúde aumenta a atividade física entre as mulheres grávidas (MALTA et al., 2016). Portanto, esses profissionais, como fornecedores de conhecimento e apoio, podem desempenhar um papel relevante na promoção de mudanças comportamentais, porém, é preciso de uma intervenção educativa nos profissionais de saúde para promover mudanças nos conhecimentos deles a respeito das diretrizes atuais sobre a prática de AF nas gestantes (MALTA et al., 2016). 
Em relação ao gasto energético (TTSP), verificou-se uma maior média no domínio doméstico e ocupacional e por último o lazer. Corroborando com este estudo, Carvalhaes et al. (2013) verificaram uma maior média do TTSP gastos pelas gestantes com atividades domésticas e uma menor média com atividades de lazer. Já Tavares et al. (2009) observaram uma maior média do TTSP com as atividades sedentárias, seguida das atividades domésticas, ocupacionais e, por último, a de lazer. Portanto, a literatura chama a atenção para o fato que a maioria das gestantes não realizam atividades de lazer. Dessa forma, esses resultados sugerem a necessidade de ações de promoção de atividade física no SUS, incentivando as atividades de lazer nessa população, como por exemplo: grupos de caminhada, aulas de ginásticas.

Outro achado neste estudo foi em relação a associação de algumas variáveis com o padrão sedentário. O trabalho fora de casa e a baixa escolaridade, por exemplo, aumentaram a probabilidade de a gestante apresentar o padrão sedentário. O estudo de Carvalhaes et al. (2013) também verificaram que o trabalho fora de casa foi apontado como possível barreira à realização de atividade física de lazer por gestantes de baixa renda provavelmente, decorrente da limitação do tempo que dispõem devido a dupla jornada.

Apesar dos resultados do estudo corroborarem com outras pesquisas. 0 estudo apresenta algumas limitações como: o uso do questionário para avaliar a atividade física. Apesar de o questionário destacar como instrumento mais acessível, de baixo custo para avaliação das AF habitual em estudo epidemiológico. O grande desafio do questionário de AF é conseguir ser sensível aos diferentes níveis de $A F$, bem como ao padrão de $A F$, para evitar erros de classificação. Principalmente, pelo fato que a faixa de classificação das gestantes em sedentárias ou ativas são mais estreitas, quando comparadas à de mulheres não grávidas. Além disso, as respostas do questionário dependem não só da memória do indivíduo, mas também da percepção subjetiva em relação ao esforço e duração das atividades cotidianas (TAKITO, NERI, BENİCIO, 2008; CHASAN-TABER et al., 2004). Outra limitação é o fato deste estudo tratar-se de uma análise secundária podendo ter alguns víeis como: de memória, entrevistador ou relacionada a seleção da amostra.

\section{CONSIDERAÇõES FINAIS}

O presente estudo teve o objetivo de verificar o padrão de atividade física de gestantes atendidas pelo SUS de uma cidade de porte médio com Índice de desenvolvimento humano alto $(0,800)$. Juntamente com o padrão de $A F$ podemos avaliar o gasto energético (TTSP) relacionado aos domínios de AF. 
Foi possível perceber que as gestantes, apesar dos esforços das organizações de saúde, elas se mantêm com pouca AF durante a gestação, principalmente, no lazer. Sendo que, as atividades domésticas são as que possuem maior gasto energético. Dessa forma, este cenário sugere a necessidade de ações de promoção de AF voltadas para este público. Estas ações podem ser de grupos de AF como caminhada, aulas de ginásticas, ioga, nas UBS de cada região, ou mesmo, o incentivo e orientação dos profissionais de saúde.

\section{AgRADECIMENTOS}

O Presente trabalho foi realizado com apoio do Conselho Nacional de Desenvolvimento Científico e Tecnológico - CNPq [processos n. ${ }^{4}$ 47221/ 2010-8 e n.o 302498/ 2015-0].

\section{Notas}

\section{CONFLITOS DE INTERESSE}

Os autores não têm conflitos de interesse, incluindo interesses financeiros específicos e relacionamentos e afiliações relevantes ao tema ou materiais discutidos no manuscrito.

\section{AUtORIA E COAUTORIA}

A autoria é responsável pelos conteúdos do texto. Os autores declaram que participaram de forma significativa na construção e formação desde estudo, tendo, enquanto autor, responsabilidade pública pelo conteúdo deste, pois, contribuíram diretamente para o conteúdo intelectual deste trabalho e satisfazem as exigências de autoria.

Carla Micheli da Silva - Concepção e desenvolvimento (desde a ideia para a investigação ou artigo, criou a hipótese); Supervisão (responsável pela organização e execução do projeto e da escrita do manuscrito); Análise / interpretação (responsável pela análise estatística, avaliação e apresentação dos resultados); Levantamento da literatura (participou da pesquisa bibliográfica e levantamento de artigos); Redação (responsável por escrever uma parte substantiva do manuscrito); Revisão crítica (responsável pela revisão do conteúdo intelectual do manuscrito antes da apresentação final).

Daniela Saes Sartoreli - Concepção e desenvolvimento (desde a ideia para a investigação ou artigo, criou a hipótese); Desenho metodológico (planejamento dos métodos para gerar os resultados); Coleta e tratamento dos dados (responsável pelos experimentos, pacientes, organização dos dados); Redação (responsável por escrever uma parte substantiva do manuscrito); Revisão crítica (responsável pela revisão do conteúdo intelectual do manuscrito antes da apresentação final). 
Dalmo Roberto Lopes Machado - Desenho metodológico (planejamento dos métodos para gerar os resultados); Redação (responsável por escrever uma parte substantiva do manuscrito); Revisão crítica (responsável pela revisão do conteúdo intelectual do manuscrito antes da apresentação final).

Gleici da Silva Casto Perdoná - Concepção e desenvolvimento (desde a ideia para a investigação ou artigo, criou a hipótese); Desenho metodológico (planejamento dos métodos para gerar os resultados); Supervisão (responsável pela organização e execução do projeto e da escrita do manuscrito); Análise / interpretação (responsável pela análise estatística, avaliação e apresentação dos resultados); Redação (responsável por escrever uma parte substantiva do manuscrito); Revisão crítica (responsável pela revisão do conteúdo intelectual do manuscrito antes da apresentação final).

\section{REFERÊNCIAS}

ACOG Committee Obstetric Practice. ACOG Committee opinion no. 267, January 2002: Exercise during pregnancy and the postpartum period American college of obstetricians and gynecologists. Obstetrics Gynecology, v. 99, n. 1, p. 171-173, jan. 2002. Disponível em: https://pubmed.ncbi.nlm.nih.gov/11777528/ Acesso em: 15 set 2017.

AINSWORTH, Barbara E.; HASKELL, William L.; WHITT, Melicia C.; IRWIN, Melinda L.; SWARTZ, Ann M.; STRATH, Scot J.; O'BRIEN, William L.; BASSETT, David R. J. R.; SCHMITZ, Kkathryn H.; EMPLAINCOURT, Patricia O.; JACOBS JR, David R.; LEON, Arthur S. Compendium of physical activities: an update of activity codes and MET intensities. Medicine \& science in sports \& exercise, v. 32, n. 9, p. S498-S516, 2000.

ARTAL, Raul; O'TOOLE, Mattew. Guidelines of the American College of Obstetricians and Gynecologists for exercise during pregnancy and the postpartum period. British journal of sports medicine, v. 37, n. 1, p. 6-12, 2003.

ASSOCIAÇÃO BRASILEIRA DE EMPRESA DE PESQUISA (ABEP). Dados com base no levantamento socioeconômico 2015 - IBOPE. São Paulo; ABEP; 23 março. 2015. Disponível em: http://www.abep.org. Acesso em: 15 set 2017.

ATALAH, Samur. Propuesta de um nuevo estándar de evaluación nutricional em embarazadas. Revista de Medicina Do Chile, v. 125, p. 1429-1436, 1997.

BARBIEIRI, Patrícia; NUNES, Juliana C.; TORRES, Alexandre G.; NISHIMURA, Renata Y.; ZUCCOLOTO, Daniela C. C.; CRIVELLENTI, Lívia C.; FRANCO, Laércio J.; SARTORELI, Daniela $\mathrm{S}$. Indices of dietary fat quality during midpregnancy is associated with gestational diabetes. Nutrition, v. 32, n. 6, p. 656-661, 2016.

BATISTA, Daniele C.; CHIARA, Vera L.; GUGELMIN, Sílvia A.; MARTINS, Patrícia D. Atividade física e gestação: saúde da gestante não atleta e crescimento fetal. Revista Brasileira de Saúde Materno Infantil, v. 3, n. 2, p. 151-158, 2003.

BICALHO, Elton de Souza. Fatores associados à prática de atividade física no primeiro trimestre em uma coorte de gestantes do Município do Rio de Janeiro. 2006. Dissertação (Mestrado em Nutrição) - Instituto de Nutrição Josué de Castro, Universidade Federal do Rio de Janeiro, 2006.

BRASIL. Ministério da Saúde. Secretaria de Políticas de Saúde. Gestação de alto risco. 3. ed. Brasília/DF, 2000. 
CARVALHAES, Maria Antonieta de Barros Leite; MARTINIANO, Ana Carolina de Almeida; MALTA, Maíra Barreto; TAKITO, Monica Yuri; BENÍCIO, Maria Helena D'Aquino. Atividade física em gestantes assistidas na atenção primária à saúde. Revista de Saúde Pública, v. 47, n. 5, p. 958-967, 2013.

CHASAN-TABER, Lisa; SCHMIDT, Michael D.; ROBERTS, Dawn E.; HOSMER, David; MARKENSON, Glenn; FREEDSON, Patty S. Development and validation of a pregnancy physical activity questionnaire. Medicine \& Science in Sports \& Exercise, v. 36, n. 10, p. 1750-1760, 2004.

DOMINGUES, Marlos Rodrigues; BARROS, Aluísio J. D. Leisure-time physical activity during pregnancy in the 2004 Pelotas Birth Cohort Study. Revista de Saúde Pública, v. 41, p. 173-180, 2007.

GUSTAVO, Andréia S. Epidemiologia da Atividade Física e sua Associação com Obesidade em Amostra Representativa da População Adulta de Porto Alegre. 2010. 149 f. Tese (Doutorado em Epidemiologia)- Faculdade de Medicina, Universidade do Rio Grande do Sul, 2010.

INSTITUTE OF MEDICINE (US). SUBCOMMITTEE ON NUTRITIONAL STATUS et al. Nutrition during pregnancy: part I, weight gain: part II, nutrient supplements. Natl Academy Pr, 1990.

KHASHAN, Ali S.; KENNY, Louise C. The effects of maternal body mass index on pregnancy outcome. European journal of epidemiology, v. 24, n. 11, p. 697, 2009.

MALTA, Maíra Barreto; CARVALHAES, Maria A. B. L.; TAKITO, Mônica; TONETE, Vera Lucia P.; BARROS, Aluíso J. D.; PARADA, Cristina M. G. L.; BENÍCIO, Maria Helena D'Aquino. Educational intervention regarding diet and physical activity for pregnant women: changes in knowledge and practices among health professionals. BMC pregnancy and childbirth, v. 16, n. 1, p. 175, 2016.

MCMILLAN, Amy Gross; MAY, Linda E.; GAINES, Georgeanna Gower; ISLER, Christy; KUEHN, Devon. Effects of Aerobic Exercise during Pregnancy on One-Month Infant Neuromotor Skills. Medicine and science in sports and exercise, v.51, n.8, p.1671-1676, 2019.

OLIVEIRA, Cibele Santini; IMAKAWA, Thiago dos Santos; MOÍSES, Elaine, DANTAS, Christine S. Physical Activity during Pregnancy: Recommendations and Assessment Tools. Revista Brasileira de Ginecologia e Obstetrícia, v. 39, p. 08, p. 424-432, 2017.

RIEBE, Deborah; ERHMAN, Jonathan K.; LIGUORI, Gary; MAGAL, MEIR (Eds.). ACSM's guidelines for exercise testing and prescription. 10. ed. Wolters Kluwer, 2018.

ROMERO, Simone Cristina S.; KURASAHIMA, Carolina H.; ROMERO, Alexandre; TAKITO, Mônica $Y$. A influência dos profissionais de saúde sobre a prática de atividade física no lazer em gestantes. Pensar a Prática, v. 18, n. 3, 2015.

SCHMIDT, Michael D.; FREEDSON, Patty S.; PEKOW, Penelope; ROBERTS, Dawn; STERNFELD, Barbara; CHASAN-TABER, Lisa. Validation of the Kaiser Physical Activity Survey in pregnant women. Medicine \& science in sports \& exercise, v. 38, n. 1, p. 4250, 2006.

SILVA, Francisco Trindade. Avaliação do Nível de Atividade Física durante a Gestação. 
2007.136 f. Dissertação (Mestrado em Saúde Pública) - Universidade Estadual do Ceará, Fortaleza, 2007. Disponível em:

http://www.uece.br/cmasp/dmdocuments/trindade 2007.PDF. Acesso em: 15 set 2017.

TAKITO, Monica Y.; BENÍCIO, Maria Helena D'Aquino; NERI, Lenycia D. C. L. Physical activity by pregnant women and outcomes for newborns: a systematic review. Revista de Saúde Pública, v. 43, n. 6, p. 59-69, 2009.

TAKITO, Monica Y.; NERI, Lenycia D. C. L.; BENÍCIO, Maria Helena D’Aquino. Avaliação da reprodutibilidade e validade de questionário de atividade física para gestantes. Revista Brasileira de Medicina do Esporte, v. 14, n. 2, p. 132-138, 2008.

TAVARES, Jousileni D. S.; MELO, Adriana S. O.; AMORIM, Melania Maria R.; BARROS, Vivianne O.; TAKITO, Mônica Y.; BENİCIO, Maria Helena D'Aquino; CARDOSO, Maria Aparecida A. Physical activity patterns in pregnant women attending the family health program of Campina Grande-PB. Revista Brasileira de Epidemiologia, v. 12, n. 1, p. 1019, 2009.

R CORE TEAM. R: A language and environment for statistical computing. 2013, Vienna, Austria: R Foundation for Statistical Computing. URL http://www. R-project. org, 2013.

WORLD HEALTH ORGANIZATION (Org.). Global recommendations on physical activity for health. World Health Organization, 2010.

Recebido em: 30 jun. 2020

Aprovado em: 05 abr. 2021

Artigo submetido ao sistema de similaridade Turnitin $®$.

A revista Conexões utiliza a Licença Internacional Creative Commons Atribuição 4.0, preservando assim, a integridade dos artigos em ambiente de acesso aberto.

A Revista Conexões é integrante do Portal de Periódicos Eletrônicos da Unicamp e associado/membro das seguintes instituições:
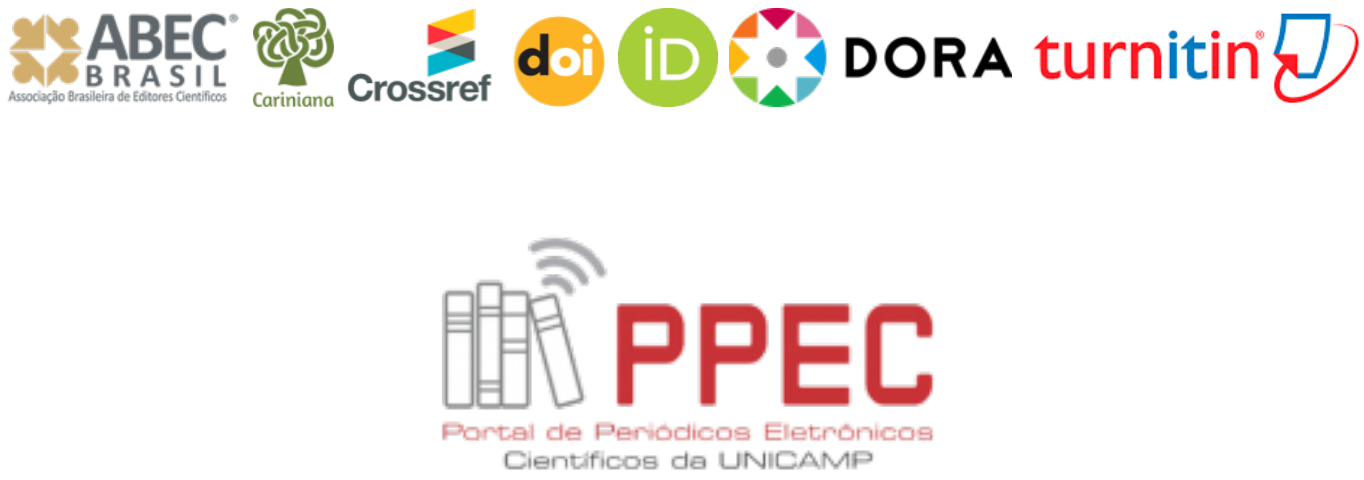